\title{
SV40T reprograms Schwann cells into stem-like cells that can re-differentiate into terminal nerve cells.
}

\section{Rui-Fang Li}

Hubei Third People's Hospital of Jianghan University

\section{Guo-Xin Nan}

Chongqing Medical University Affiliated Children's Hospital

\section{Dan Wang}

Hubei Third People Hospital of Jianghan University

\section{Chang Gao}

Hubei Third People's Hospital of Jianghan University

\section{Juan Yang}

Hubei Third People's Hospital of Jianghan University

\section{Tong-Chuan He}

University of Chicago Department of Medicine

\section{Zhong-lin Zhang ( $\boldsymbol{2}$ 2645176131@qq.com )}

Wuhan University Zhongnan Hospital

\section{Research article}

Keywords: Schwann cells, SV40T, cell transformation, cell reprogramming, Inverse differentiation, neurocrest cells

Posted Date: March 18th, 2020

DOI: https://doi.org/10.21203/rs.3.rs-17698/v1

License: (c) (1) This work is licensed under a Creative Commons Attribution 4.0 International License. Read Full License

Version of Record: A version of this preprint was published at The International Journal of Developmental Biology on January 1st, 2021. See the published version at https://doi.org/10.1387/ijdb.210062zz. 
${ }^{*}$ Correspondence:

zhonglinzhang@whu.edu.cn

${ }^{4}$ Department of Hepatobiliary

Surgery, the Research Center of

Digestive Diseases, Zhongnan

Hospital of Wuhan University,

Donghu Road 169, 430000

Wuhan, China

Full list of author information is available at the end of the article

$\dagger$ Equal contributor,

tche@uchicago.edu

\begin{abstract}
Background: The effects of Simian virus $40 \mathrm{~T}$ antigen (SV40T) on various kinds of cells are different. Previous researchers failed to use SV40T immortalized nerve cells. However, they argued that SV40T caused nerve cell transformation. No one further study what is the specific effect of SV40T on nerve cells. We transfected Schwann cells (SCs) that did not have differentiation ability with MPH 86 plasmid containing SV40T in order to explore the effects of SV40T on Schwann cells.
\end{abstract}

Methods: SCs were transfected with MPH 86 plasmid carrying the SV40T gene and cultured in different media, as well as co-cultured with neural stem cells (NSCs). In our study, SCs overexpressing SV40T were defined as SV40T-SCs. The proliferation of these cells was detected by WST-1, and the expression of different biomarkers was analyzed by qPCR and immunohistochemistry.

Results: SV40T induced the characteristics of NSCs, such as the ability to grow in suspension, form spheroid colonies and proliferate rapidly, in the SCs, which were reversed by knocking out SV40T by the Flip-adenovirus. In addition, SV40T up-regulated the neurocrest markers Nestin, Pax3 and Slug, and down-regulated $\mathrm{S} 100 \mathrm{~b}$ as well as the late differentiation markers MBP, GFAP and Olig1/2. These cells also expressed NSC markers like Nestin, Sox2, CD133 and SSEA-1, as well as early development markers of embryonic stem cells (ESCs) like BMP4, c-Myc, OCT4 and Gbx2. Co-culturing with NSCs induced differentiation of the SV40T-SCs into neuronal and glial cells.

Conclusions: SV40T reprograms Schwann cells to stem-like cells at the stage of neural crest cells that can differentiate to terminal nerve cells.

Keywords: Schwann cells; SV40T; cell transformation; cell reprogramming; Inverse differentiation; neurocrest cells

\section{Background}

Reprogramming, or the de-differentiation of mature cells to the totipotent stem-cell like state via genetic or epigenetic mediation [1], is a potent tool for achieving tissue engineering and repair in various degenerative diseases, especially neurological disorders. Schwann cells (SCs) are glial cells that have the ability to repair damaged peripheral nerve myelin sheath $[2,3]$, and can theoretically be reprogramed for potential clinical applications. Although the Simian virus $40 \mathrm{~T}$ antigen (SV40T)SV40T is routinely used to construct immortalized cell lines [4], it can induce cell transformation depending on the host cell type [5]. For example, forced expression of 
SV40T in brain tissues leads to the loss of terminal differentiation markers on the astrocytes, and the cells acquire an early differentiated phenotype [6]. Moreover, Mali et al found that SV40T significantly improved reprogramming efficiency [7].

We constructed mouse sciatic nerve SCs by transfecting the primary cells with the MPH 86 plasmid [5] carrying the SV40T gene. We found SV40T induced the expression of Sox 2 and c-Myc that are the important transcription factors that have a role in reprogramming of somatic cells [8]. Reprograming induces differentiated cells into reverting to stem-like cells [9]. The ensuing SV40T-overexpressing SCs showed significant changes in their morphology, proliferation rates, phenotypic markers etc. compared to primary SCs. These characteristics of SV40T-overexpressing SCs are similar to stem-like cells at the stage of neural crest cells. Based on our findings, we surmised that SV40T-mediated reprogramming led to the reverse differentiation of SCs.

\section{Methods}

\section{Cell culture}

Primary SCs were obtained from ATCC (Manassas, VA). Primary SCs were cultured in serum free Neurobasal medium supplemented with B27. Neural stem cells (NSCs) derived from rat hippocampus gyrus were cultured in DMEM/F12 N2 medium supplemented with B27, 20ng/ml hFGF2, 0.1mM $\beta$-ME, $100 \mathrm{IU} / \mathrm{ml}$ Penicillin and $100 \mathrm{ug} / \mathrm{ml}$ Streptomycin. Unless indicated otherwise, all chemicals were purchased from either Sigma-Aldrich (St. Louis, MO) or Thermo Fisher Scientific (Pittsburgh, $\mathrm{PA})$.

Primary SCs were transfected with MPH 86 plasmid carrying the SV40T and Hygromycin resistance genes (Figure 1A) using Lipofectamine as per the manufacturer's instructions. The stably transfected cells were screened using 100 $\mu \mathrm{g} / \mathrm{ml}$ Hygromycin, and the drug-resistant clones were further expanded. SV40Toverexpressing cells were confirmed by T-D PCR, and transduced with the Flip adenovirus (Ad-Flip) or Ad-GFP. The expression of SV40T was detected by T-D PCR 24h after viral infection. In our study, SCs overexpressing SV40T were defined as SV40T-SCs.

\section{Proliferation assay}

The SV40T-SCs were cultured in both serum-free Neurobasal medium and the $10 \%$ FBS-supplemented NSC culture medium, and their morphology and growth rates were recorded. In addition, cells transduced with Ad-Flip and Ad-GFP were cultured in complete DMEM for one week, and the proliferation rates were analyzed by crystal violet staining and WST-1 $[5,10]$. The growing cells were plated into 96well culture plates at $20 \%$ confluence. Wells without seeding any cells were used as background controls. At the indicated time points, $4 \mathrm{ml}$ of the premixed WST-1 (BD Clontech, Mountain View, CA) were added to each well and incubated at the $37{ }^{\circ} \mathrm{C} \mathrm{CO}_{2}$ incubator for $1 \mathrm{hr}$. $100 \mu \mathrm{gl}$ of the WST-1-containing culture medium were taken for absorbance reading at $440 \mathrm{~nm}$ using a plate reader. The obtained A44 $\mathrm{nm}$ values were subjected to background reading subtractions. Each assay condition was done in triplicate. 
RNA isolation and quantitative real-time PCR (qPCR)

Total RNA was isolated using TRIZOL reagent (Life Technologies) and reverse transcribed using hexamer primers and M-MuLV reverse transcriptase (New England Biolabs, Ipswich, MA). The qPCR primers (Additional file 1) were designed with the Primer 3 program. SYBR Green-based qPCR analysis was performed using CFX-96 Connect (Bio-Rad, CA). All samples were tested in triplicate and the standard sample provided in the kit was used as the negative control.

Immunofluorescence

The SV40T-SCs cultured in 24-well plates, fixed with methanol, permeabilized with $1 \%$ NP-40, and blocked with $10 \%$ donkey or goat serum. The cells were then incubated with antibodies against Nestin, CD133, SSEA-1, Sox2, Oct4, c-Myc, Gbx2 and BMP4, washed, and probed with Texas Red or Green-labeled secondary antibody as appropriate. After counterstaining with DAPI, the slides were examined under a fluorescence microscope.

SCs and rat hippocampal gyrus NSCs co-culture

The SV40T-SCs and NSCs were respectively transfected with RFP and GFP using the piggybac transposon vector system. The cells were mixed at equal densities $\left(1 \times 10^{6}\right)$ in $100 \mu \mathrm{l}$ complete DMEM and seeded into 6-well plates pre-coated with rat tail glue. The cells were co-cultured for 1-2 weeks, and the morphological changes were observed under a fluorescence microscope. On day 7 of culture, the cells were harvested, sorted by flow cytometry and re-seeded into 24-well plates. After 24h, the cells were fixed with methanol, and the expression of Nestin, S100b and beta-III tubulin was detected by immunohistochemistry [11].

\section{Results}

SV40T reprogrammed the SCs into NSC-like cells

The SCs transfected with MPH 86 plasmid carrying the SV40T stably expressed SV40T gene (Figure 1B). As shown in Figure 1B, SV40T expression was significantly decreased in the Ad-Flip group. Furthermore, SV40T knockdown significantly decreased the proliferation rates of cells compared to that of Ad-GFP-infected SCs (Figure 1C and 1D).

The primary SCs were adherent in Neurobasal medium containing B27, and showed the long synapses characteristic of nerve cells, along with a slow growth rate (Figure 2A). However, the SCs did not proliferate in complete DMEM. In contrast, SV40T-SCs grew rapidly in both media. Complete DMEM resulted in adherent growth with short synapses, round, triangular, diamond-shaped or striped morphologies (Figure 2B), and formation of spherical colonies (Figure 2C). In the NSC medium however, these cells grew in suspension attaining a round shape, and formed spherical colonies (Figure 2D and 2E). The NSCs were used as controls (Figure 2F). Thus, forced expression of SV40T induced characteristics of stem cells in the primary SCs.

Consistent with the phenotypic changes, SV40T increased the expression levels of the neural crest markers Slug and Pax3, as well as the NSC marker Nestin in the SCs. In contrast, the SC marker S100b [12], and the later neuronal differentiation 
markers MBP, GFAP and Olig1/2 were weakly expressed in these cells (Figure 3). Furthermore, the SV40T-SCs were positive for the NSC markers Nestin, Sox2, CD133 and SSEA-1, as well as the embryonic stem cell (ESC) markers BMP4, cMyc, OCT4 and Gbx2 (Figure 4). Taken together, SV40T reprograms the primary SCs to the early stages of neuronal differentiation.

SV40T-SCs re-differentiate into neuronal and glial cells following co-culture with NSCs The SV40T-SCs were labeled with RFP (Figure 5A), and the NSCs with GFP (Figure 5B). After 7 days of co-culture in 10\% FBS-supplemented DMEM, the SCs showed significantly elongated synapses, which were intertwined with that of the NSCs (Figures 5I). Nestin, beta-III tubulin (neuron cell marker) and S100b (Schwann cell marker) also up-regulated in the co-cultured SCs, and these cells corresponded to neuron-like cells, neuronal morphology with triangular shape and long axons, and SC morphology (Figure 6). Thus, the SV40T-SCs can differentiate into neurons and glial cells.

\section{Discussion}

To determine the biological role of SV40T in the primary SCs, we transfected the cells with MPH86 plasmid carrying the SV40T gene. The transgenic cells grew rapidly in the NSC medium and complete DMEM, and formed spheroid colonies in suspension. In addition, SV40T significantly up-regulated Nestin, Pax3 and Slug, which are the major markers of NSCs and neural crest cells. However, the SC marker S100b, as well as the late neuronal markers MBP, GFAP and Olig1/2, were markedly lower following SV40T expression. Furthermore, SV40T also induced various NSC and ESC-related factors like Nestin, Sox2, CD133, SSEA-1, BMP4, c-Myc, OCT4 and Gbx2. The Sox2 and c-Myc are the important transcription factors that have a role in reprogramming of somatic cells [8]. We surmised therefore that SV40T reprograms the SCs to stem-like cells [9]. To test this hypothesis, we co-cultured the SCs with NSCs, and observed a significant lengthening and intertwining of the synapses, along with other morphological and immune-phenotypic characteristics of various nerve cells. Therefore, the SV40T-SCs are possibly stem-like cells at the stage of neural crest cells that can differentiate into the terminal neural cells.

During embryonic development, the notochord and adjacent mesenchymal cells induce the ectoderm on its surface to form a neural plate, and its central axis forms a depression known as the nerve groove or fold. The junction of the top of this fold and the peripheral ectoderm is called neural crest. The neural crest cells are pluripotent stem cells that arise from the triploblasts in the third week of embryonic development, and differentiate into multiple cell types that migrate into the mesoderm once the neural tube closes [13]. The migration of neural crest cells depends on Slug and RhoB that are induced by Wnt, FGF, BMP4 and BMP7. In addition, epithelial mesenchymal transition (EMT), or the differentiation from the ectoderm into mesoderm, is also critical for the migration and differentiation of neural crest cells, and is controlled by PAX3, which plays a key role in the development of embryonic nerve cells. PAX3 and Msx1 induce Snail1 and Snail2 to down-regulate the expression of adhesion proteins, thereby allowing the neural crest cells to migrate and differentiate into mesenchymal cells [14]. The transcription 
factor GBX2 antagonizes OTX2, which inhibits PAX6 expression and induces the production of post-brain neural progenitor cells. Sox 2 gene is widely expressed in the neural endoderm at the beginning of the formation of gut embryo, and is induced by chordin to promote the differentiation of nerve cells [6]. We detected high levels of the above factors in the SV40T-SCs, which supports our aforementioned hypothesis. The differentiation potential of these cells need to be verified by further experiments.

SV40T functions as an ATPase and DNA helicase, and is also involved in the phosphorylation of protein serine/threonine residues, and ribosylation and acylation of ADP. SV40T also activates ribosomal genes in host cells, induces DNA synthesis, and modifies initiation factors of protein synthesis. Previous studies have suggested a key role of SV40T in oncogenic transformation as well [15]. SV40T alters cell cycle and proliferation by inhibiting the expression of $\mathrm{p} 53$ and RB proteins [1]. It forms a complex with and inactivates p53 protein [16], which is known to promote MDM2-mediated ubiquitination and degradation of Slug [17]. The latter is essential for stem cell maintenance and de-differentiation. Therefore, SV40T regulates cell cycle through p53 and inhibits p53-mediated degradation of Slug, which down-regulates E-cadherin and induces EMT [18]. Oct4, Sox2 and c-myc induce cellular reprogramming [1], and were upregulated in the SV40T SCs. Previous studies have shown that SV40 T can improve reprogramming efficiency $[7,19]$. The reprogramming of Schwann cells by SV40T may involve cell cycle regulation and Slug activation.

\title{
Conclusion
}

SV40T reprograms Schwann cells to stem-like cells at the stage of neural crest cells that can differentiate to terminal nerve cells.

\author{
Additional Files \\ Additional file 1 \\ Table S1. presenting sequences of primers used. \\ Abbreviations \\ SCs: Schwann cells; NSCs: neural stem cells; ESCs: embryonic stem cells; SV40T: Simian virus 40 T antigen; EMT: \\ epithelial mesenchymal transition. \\ Acknowledgements \\ None. \\ Funding \\ This work is supported by the Health Commission of Hubei Province (WJ2017M150; WJ2019M209). \\ Availability of data and materials \\ All data generated or analyzed during this study are included in this published article and its Additional file 1. \\ Author's contributions \\ ZL-Zhang and TC-He conceived, designed, and supervised all aspects of the project with input from RF-Li. RF-Li \\ performed most experiments. GX-Nan performed, analyzed, and wrote the cell culture experiments. D-Wang, C-Gao \\ and J-Yang participated in writing the manuscript. RF-Li, TC-He and ZL-Zhang wrote the manuscript with input \\ from all authors. All authors read and approved the final manuscript. \\ Author's information \\ Not available. \\ Ethics approval and consent to participate \\ Not available. \\ Competing interests \\ Not available.
}


Competing interests

The authors declare that they have no competing interests.

Author details

${ }^{1}$ Department of Neurology, Hubei NO.3 People's Hospital of Jianghan University, Zhongshan Avenue 26, Wuhan, China. ${ }^{2}$ Molecular Oncology Laboratory, Department of Orthopaedic Surgery and Rehabilitation Medicine, The University of Chicago Medical Center, Zhongshan Second Road 136, 400014 Chongqing, China. ${ }^{3}$ Molecular Oncology Laboratory, Department of Orthopaedic Surgery and Rehabilitation Medicine, The University of Chicago Medical Center, South Maryland Avenue 5841, 60601 Chicago, USA. ${ }^{4}$ Department of Hepatobiliary Surgery, the Research Center of Digestive Diseases, Zhongnan Hospital of Wuhan University, Donghu Road 169, 430000 Wuhan, China.

\section{References}

1. Takahashi, K., Yamanaka, S.: Induction of pluripotent stem cells from mouse embryonic and adult fibroblast cultures by defined factors. Cell 126(4), 663-76 (2006). doi:10.1016/j.cell.2006.07.024

2. Hyung, S., Jung, K., Cho, S.R., Jeon, N.L.: The schwann cell as an active synaptic partner. Chemphyschem 19(10), 1123-27 (2018). doi:10.1002/cphc.201701299

3. Baron-Van Evercooren, A., Avellana-Adalid, V., Lachapelle, F., Liblau, R.: Schwann cell transplantation and myelin repair of the cns. Mult Scler 3(2), 157-61 (1997). doi:10.1177/135245859700300219

4. Kaiser, B., Bottner, M., Wedel, T., Brunner, R.M., Goldammer, T., Lesko, S., Gabel, G., Gleich, A., Pfannkuche, H.: Establishment and characterization of an sv40 large $t$ antigen-transduced porcine colonic epithelial cell line. Cells Tissues Organs 203(5), 267-286 (2017). doi:10.1159/000453394

5. Wang, N., Zhang, W., Cui, J., Zhang, H., Chen, X., Li, R., Wu, N., Chen, X., Wen, S., Zhang, J., Yin, L., Deng, F., Liao, Z., Zhang, Z., Zhang, Q., Yan, Z., Liu, W., Ye, J., Deng, Y., Wang, Z., Qiao, M., Luu, H.H. Haydon, R.C., Shi, L.L., Liang, H., He, T.C.: The piggybac transposon-mediated expression of sv40 $t$ antigen efficiently immortalizes mouse embryonic fibroblasts (mefs). PLoS One 9(5), 97316 (2014) doi:10.1371/journal.pone.0097316

6. Stevanovic, M., Lovell-Badge, R., Collignon, J., Goodfellow, P.N.: Sox3 is an x-linked gene related to sry. Hum Mol Genet 2(12), 2013-8 (1993). doi:10.1093/hmg/2.12.2013

7. Mali, P., Ye, Z., Hommond, H.H., Yu, X., Lin, J., Chen, G., Zou, J., Cheng, L.: Improved efficiency and pace of generating induced pluripotent stem cells from human adult and fetal fibroblasts. Stem Cells 26(8), 1998-2005 (2008). doi:10.1634/stemcells.2008-0346

8. Yamanaka, S.: Strategies and new developments in the generation of patient-specific pluripotent stem cells. Cell Stem Cell 1(1), 0-49 (2017). doi:10.1016/j.stem.2007.05.012

9. Jopling, C., Boue, S., Belmonte, J.C.I.: Dedifferentiation, transdifferentiation and reprogramming: three routes to regeneration. Nature Reviews Molecular Cell Biology 12(2), 79-89 (2011). doi:10.1038/nrm3043

10. He, B.-C., Chen, L., Zuo, G.-W., Zhang, W., Bi, Y., Huang, J., Wang, Y., Jiang, W., Luo, Q., Shi, Q., Zhang, B.-Q., Liu, B., Lei, X., Luo, J., Luo, X., Wagner, E.R., Kim, S.H., He, C.J., Hu, Y., Shen, J., Zhou, Q., Rastegar, F., Deng, Z.-L., Luu, H.H., He, T.-C., Haydon, R.C.: Synergistic antitumor effect of the activated ppargamma and retinoid receptors on human osteosarcoma. Clinical cancer research : an official journal of the American Association for Cancer Research 16(8), 2235-2245 (2010). doi:10.1158/1078-0432.CCR-09-2499

11. Huang, J., Bi, Y., Zhu, G.-H., He, Y., Su, Y., He, B.-C., Wang, Y., Kang, Q., Chen, L., Zuo, G.-W., Luo, Q., Shi, Q., Zhang, B.-Q., Huang, A., Zhou, L., Feng, T., Luu, H.H., Haydon, R.C., He, T.-C., Tang, N.: Retinoic acid signalling induces the differentiation of mouse fetal liver-derived hepatic progenitor cells. Liver international : official journal of the International Association for the Study of the Liver 29(10), 1569-1581 (2009). doi:10.1111/j.1478-3231.2009.02111.x

12. Shen, M., Tang, W., Cao, Z., Cao, X., Ding, F.: Isolation of rat schwann cells based on cell sorting. Mol Med Rep 16(2), 1747-1752 (2017). doi:10.3892/mmr.2017.6777

13. O'Rahilly, R., Müller, F.: The development of the neural crest in the human. Journal of anatomy 211(3), 335-351 (2007)

14. Kim, H., Ankamreddy, H., Lee, D.J., Kong, K.-A., Ko, H.W., Kim, M.H., Bok, J.: Pax3 function is required specifically for inner ear structures with melanogenic fates. Biochemical and biophysical research communications 445(3), 608-614 (2014). doi:10.1016/j.bbrc.2014.02.047

15. Moens, U., Van Ghelue, M., Kristoffersen, A.K., Johansen, B., Rekvig, O.P., Degré, M., Rollag, H.: Simian virus 40 large t-antigen, but not small t-antigen, trans-activates the human cytomegalovirus major immediate early promoter. Virus genes 23(2), 215-226 (2001)

16. Dobbelstein, M., Roth, J.: The large $t$ antigen of simian virus 40 binds and inactivates p53 but not $p 73$. The Journal of general virology 79 ( Pt 12), 3079-3083 (1998)

17. Wang, S.P., Wang, W.L., Chang, Y.L., Wu, C.T., Chao, Y.C., Kao, S.H., Yuan, A., Lin, C.W., Yang, S.C., Chan, W.K., Li, K.C., Hong, T.M., Yang, P.C.: p53 controls cancer cell invasion by inducing the mdm2-mediated degradation of slug. Nat Cell Biol 11(6), 694-704 (2009). doi:10.1038/ncb1875

18. Leong, K.G., Niessen, K., Kulic, I., Raouf, A., Eaves, C., Pollet, I., Karsan, A.: Jagged1-mediated notch activation induces epithelial-to-mesenchymal transition through slug-induced repression of e-cadherin. J Exp Med 204(12), 2935-48 (2007). doi:10.1084/jem.20071082

19. Tan, L., Ke, Z., Tombline, G., Macoretta, N., Hayes, K., Tian, X., Lv, R., Ablaeva, J., Gilbert, M., Bhanu, N.V., Yuan, Z.F., Garcia, B.A., Shi, Y.G., Shi, Y., Seluanov, A., Gorbunova, V.: Naked mole rat cells have a stable epigenome that resists ipsc reprogramming. Stem Cell Reports 9(5), 1721-1734 (2017). doi:10.1016/j.stemcr.2017.10.001 


\section{Figures}

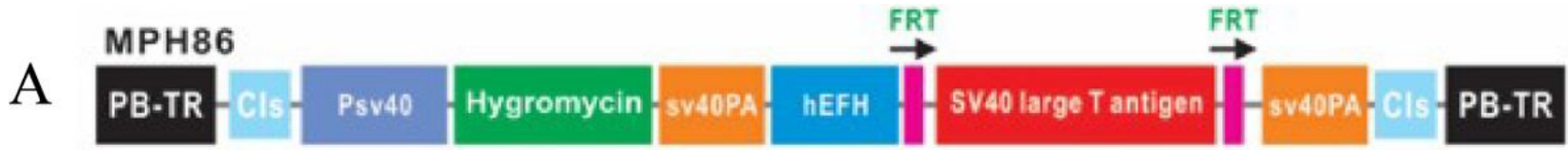 \\ PB-TR=piggyBac terminal repeat \\ $\mathrm{Cl}=$ core insulators \\ FRT=FIp recognition traget site}

B

SV40T

GAPDH
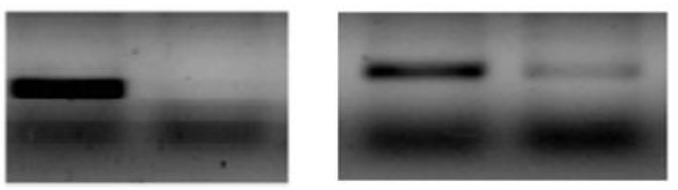

SV40T-SCs SCs

Ad-GFP Ad-Flip

$\mathrm{C}$

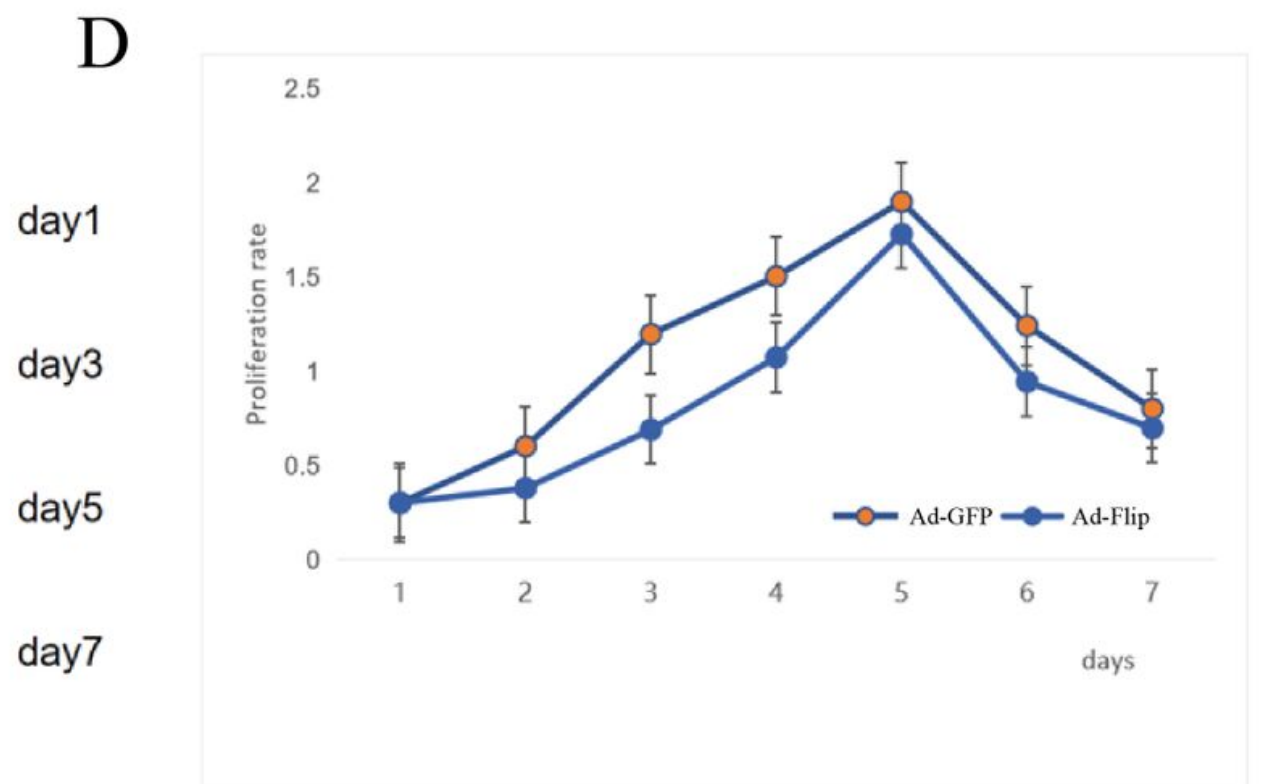

\section{Figure 1}

The SCs were transfected with SV40T and exhibited higher proliferative activity than that of primary SCs. Representative images showing (A) MPH86 plasmid structure, (B) Expression of SV40T in SCs, SV40TSCs and cells in the Ad-Flip and Ad-GFP groups, (C) Cells in the Ad-Flip and Ad-GFP groups were stained with crystal violet at the indicated time points, (D) Percentage of proliferating cells in the Ad-Flip and AdGFP groups. 


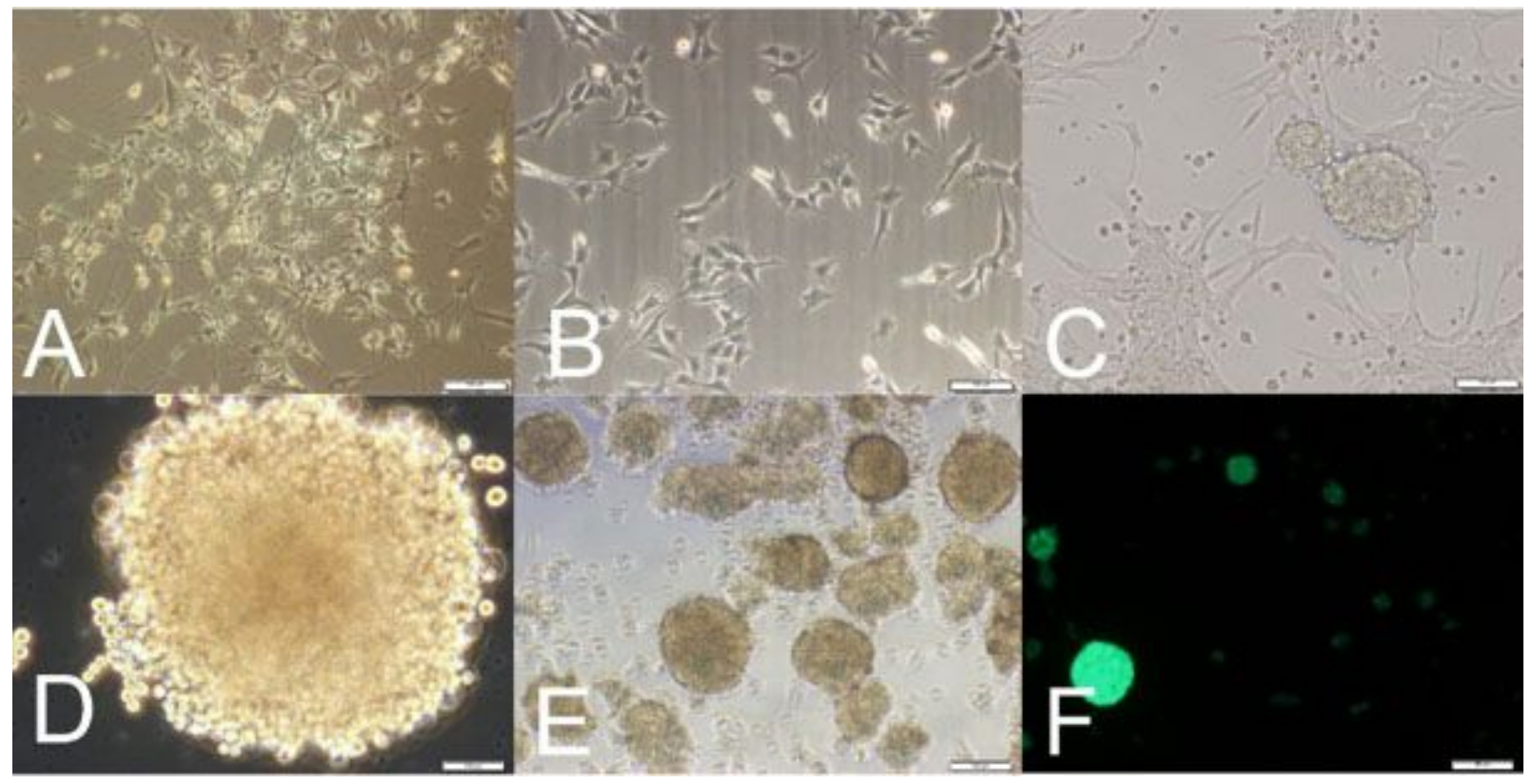

\section{Figure 2}

SV40T-SCs grow with stem cell-like growth characteristics. Representative images showing (A) primary SCs forming long synapses in NSC medium (magnication 100x), (B) adherent growth of SV40T-SCs with short synapses in complete DMEM (magnication 200x), (C) spherical colonies of SV40T-SCs in suspension in complete DMEM (magnication 200x), (D) a round shape, and formed spherical colony of SV40T-SCs in suspension (magnication 400x), (E) round shape, and formed spherical colonies of SV40TSCs grown in suspension in NSC medium (magnication 200x), and (F) NSCs grown in suspension in NSC medium (magnication 200x). 


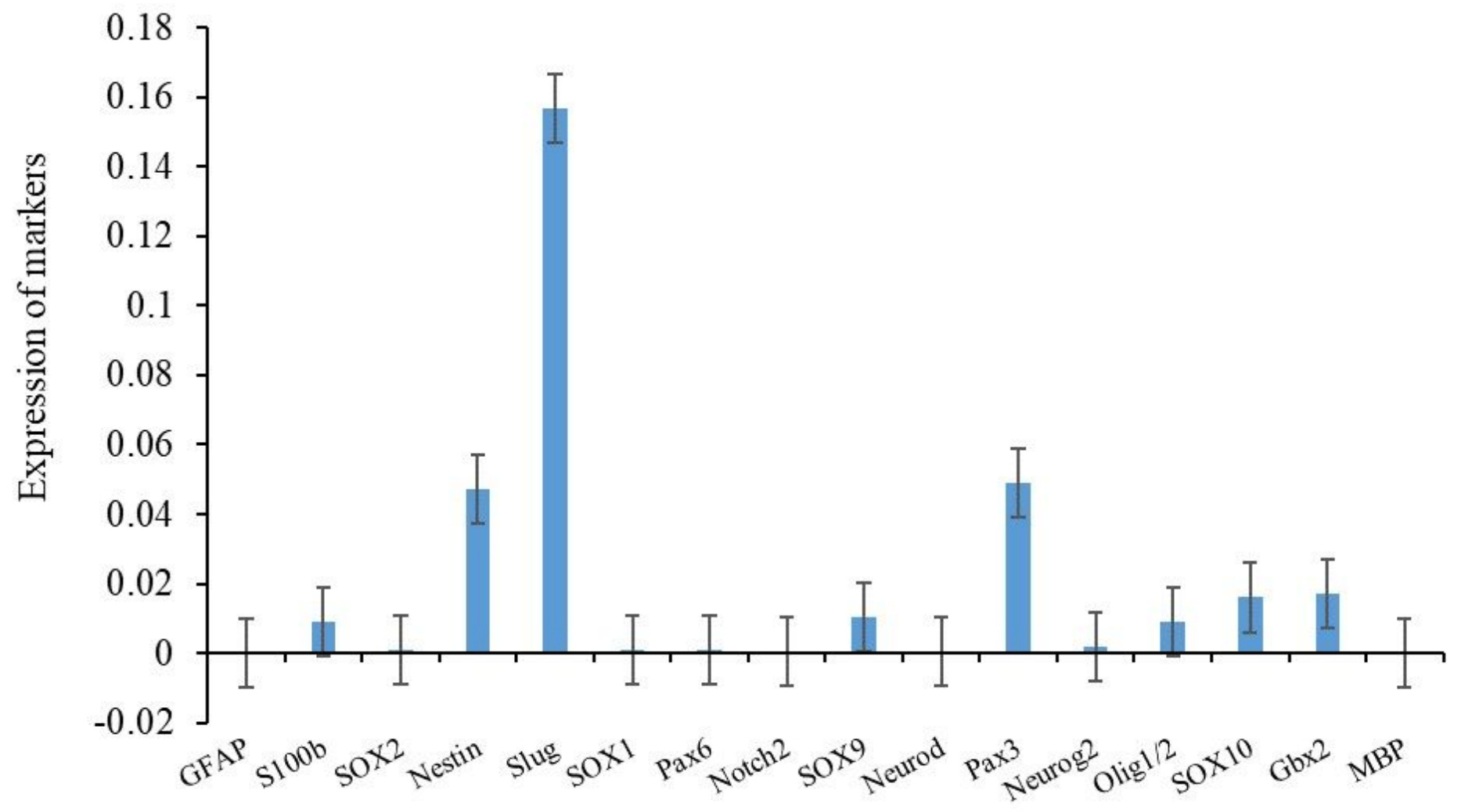

Figure 3

The expression level of the neural crest markers and the NSC marker in SV40T-SCs. SV40T-SCs highly expressed Nestin, Slug and Pax3.

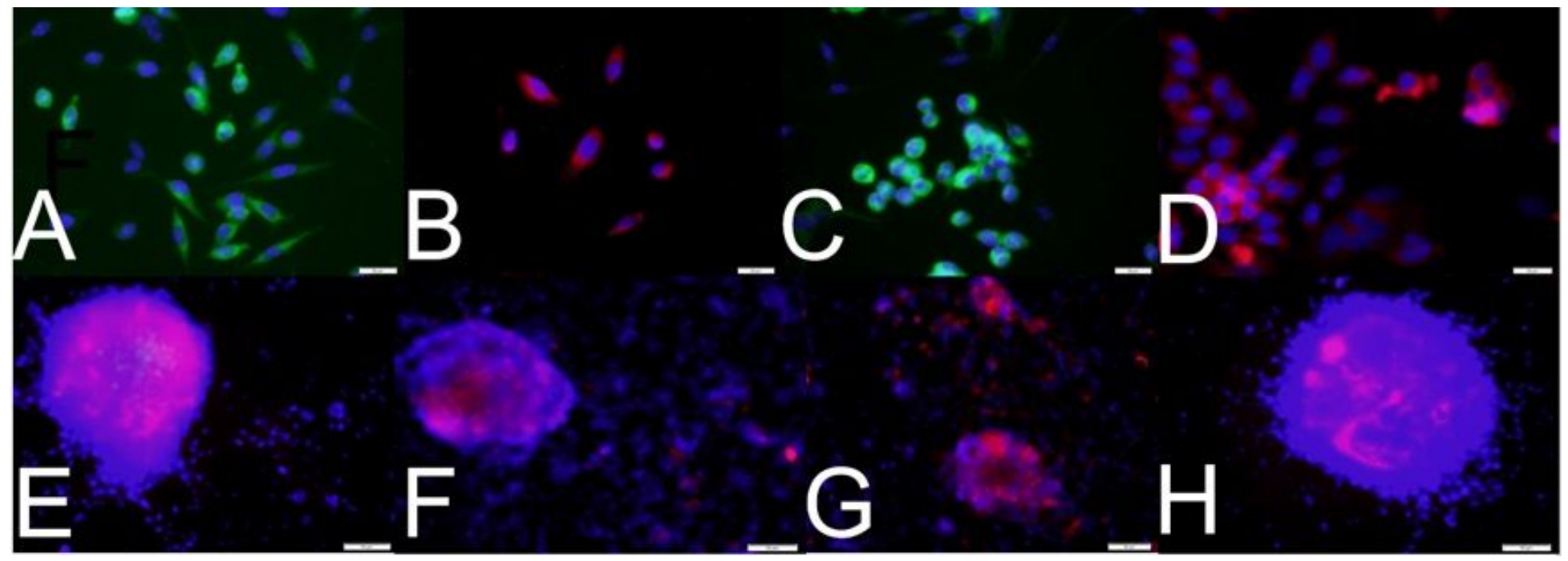

Figure 4

SV40T-SCs expressed markers of early neural diðerentiation. Representative images showing expression of Nestin (A), Sox2 (B), CD133 (C), SSEA-1 (D), BMP4 (E), c-Myc (F), OCT4 (G), and Gbx2 (H) (magnication 200x). 


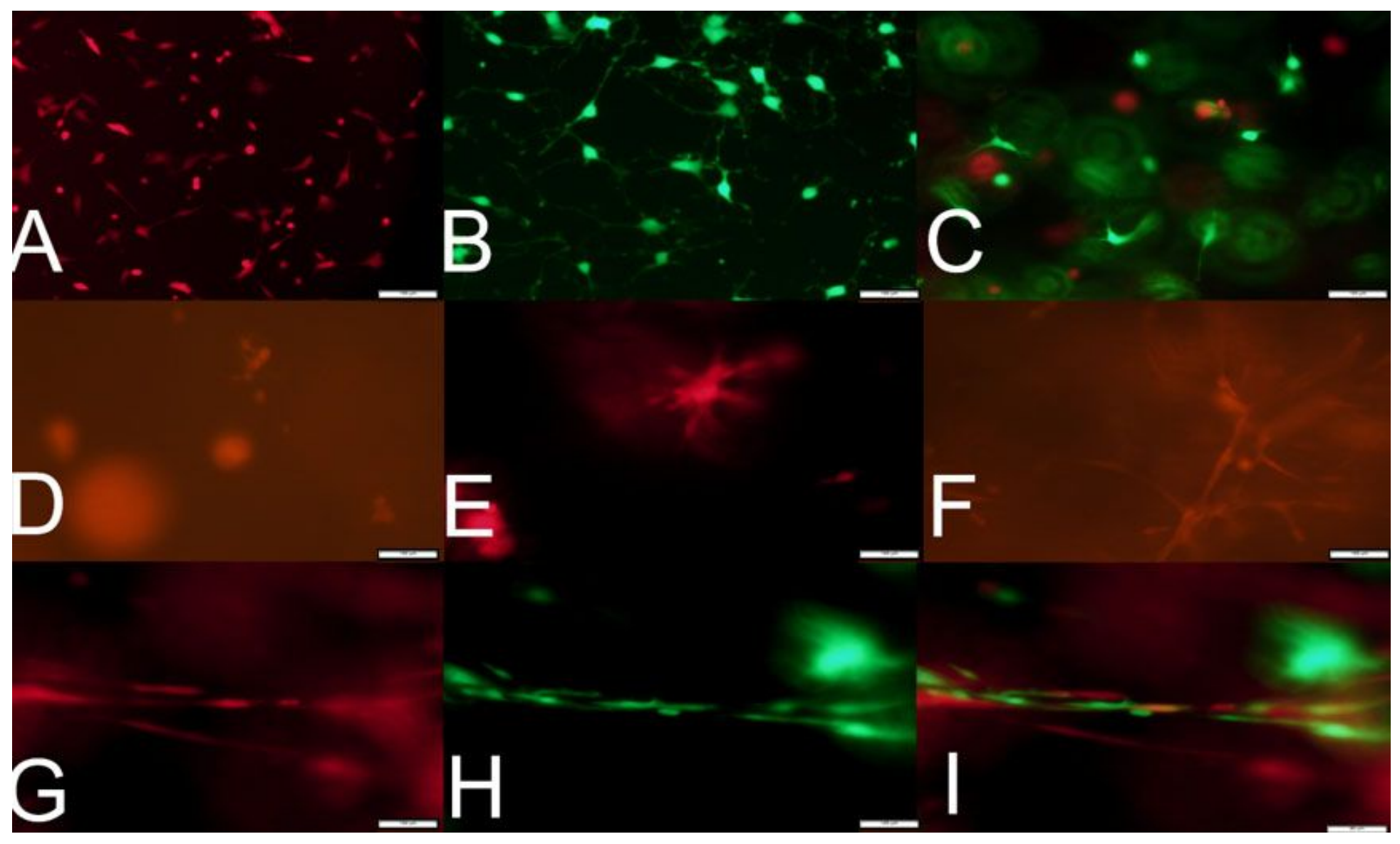

\section{Figure 5}

SV40T-SCs di囚erentiated to neuronal and glial cells in the presence of NSCs. Representative images showing (A) RFP-labeled SV40T-SCs in complete DMEM (magnication 100x), (B) GFP-labeled NSCs and in complete DMEM (magnication 100x), (C) co-cultured SV40T-SCs and NSCs on day 1 (magnication 100x), (D-G) RFP-labeled SV40T-SCs on days 1 (D), 3 (E), 5 (F) and 7 (G) of co-culture with NSCs (magnication 200x), (H) GFP-labeled NSCs on day 7 of co-culture with SV40T-SCs (magnication 400x), and (I) the merging of G and I showed intertwined synapses of SV40T-SCs and NSCs on day 7 of coculture (magnication 400x).

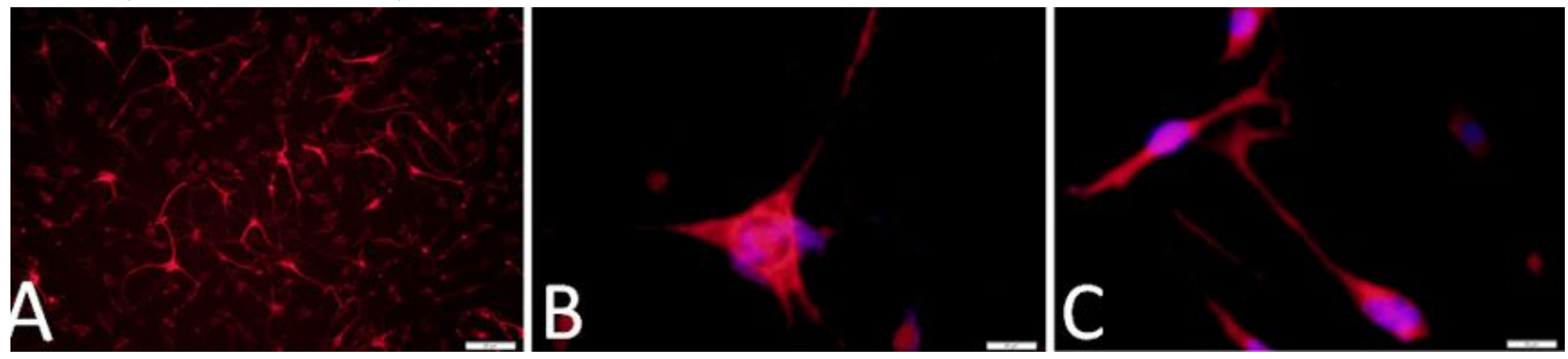

\section{Figure 6}

NSCs co-culture induces neuronal di囚erentiation in SV40T-SCs. Representative images showing expression levels of (A) Nestin (magnication 100x), (B) -IIl tubulin (magnication 400x), and (C) S100b 
(magnication 400x) in the co-cultured SV40T-SCs.

\section{Supplementary Files}

This is a list of supplementary files associated with this preprint. Click to download.

- Additionalfile1.docx 